\title{
DOCUMENT
}

DE TRAVAIL

$\mathrm{N}^{\circ} 236$

\section{VARIANTES EN UNIVERS INCERTAIN}

Stéphane Adjemian, Christophe Cahn, Antoine Devulder et Nicolas Maggiar

\section{BANQUE DE FRANCE}

EUROSYSTÈME 


\section{VARIANTES EN UNIVERS INCERTAIN}

Stéphane Adjemian, Christophe Cahn, Antoine Devulder et Nicolas Maggiar

Juin 2009

Les Documents de Travail reflètent les idées personnelles de leurs auteurs et n'expriment pas nécessairement la position de la Banque de France. Ce document est disponible sur le site internet de la Banque de France « www.banque-france.fr $»$.

Working Papers reflect the opinions of the authors and do not necessarily express the views of the Banque de France. This document is available on the Banque de France Website "www.banque-france.fr". 


\title{
Variantes en Univers Incertain*
}

\author{
Stéphane Adjemian \\ Christophe Cahn \\ Université du Maine, GAINS et CEPREMAP \\ Banque de France, DAMEP \\ Antoine Devulder \\ Nicolas Maggiar \\ Banque de France, DAMEP \\ Banque de France, DAMEP
}

* Cette étude s'inscrit dans le cadre d'un programme de travail développé à la Banque de France. Nous remercions Gilbert Cette, Olivier de Bandt, Jean-Pierre Villetelle et Jean-Pierre Laffargue pour leurs commentaires. Les opinions exprimées ici n'engagent que les auteurs, et pas les institutions auxquelles ils sont affiliés. 


\title{
Résumé
}

Nous proposons d'illustrer l'intérêt de l'approche bayésienne dans le cadre de l'évaluation des politiques économiques, réalisée le plus souvent à l'aide de variantes. Nous présentons un modèle d'équilibre général stochastique dynamique (DSGE) pour la zone euro. L'estimation bayésienne de ce modèle mesure l'incertitude sur les paramètres, qui se traduit en une incertitude sur les variantes. Nous donnons une application pratique en simulant les effets d'une politique fiscale (choc de TVA annoncé).

Classification JEL: E4, E5.

Mots-clés: DSGE, zone euro, rigidités nominales, estimation bayesienne.

\begin{abstract}
In this paper, we try to illustrate the interest of the Bayesian approach for the evaluation of economic policies, often realised by analysing the response of the economy to a standard shock. We present a Stochastic Dynamic General Equilibrium model for the euro area. The Bayesian estimation gives a measure of the uncertainty on the parameters, from which we can derive the uncertainty of the responses to standard shocks. As an illustration, we simulate the effects of a fiscal shock (announced VAT increase).
\end{abstract}

JEL Classification: E4, E5.

Keywords: DSGE, euro zone, nominal rigidities, bayesian estimation. 


\section{Résumé non technique}

Les modèles d'équilibre général intertemporels stochastiques (MEGIS, ou DSGE en anglais) sont fondés sur l'optimisation du comportement des agents économiques, en fonction de l'information qui est à leur disposition. En particulier, leurs choix tiennent compte des anticipations qu'ils font sur l'état futur de l'économie. Cette propriété explique en grande partie que de nombreuses institutions en charge de la politique économique tendent à développer ce type de modèle, en plus des modèles macroéconométriques habituellement utilisés. Leurs fondements microéconomiques permettent en effet des interprétations réalistes de simulations de politiques économiques. En outre, la prise en compte des anticipations des agents permet de s'interroger sur les impacts de l'annonce d'une politique économique : comment les agents modifieront-ils leurs choix, et quelles en seront les conséquences sur les principales variables macroéconomiques?

Pour illustrer la manière dont les anticipations sont prises en compte, nous nous intéressons dans cet article aux impacts d'une modification permanente et annoncée d'une politique fiscale future. Plus précisément, nous analysons les effets de l'annonce d'une augmentation de deux points du taux de TVA à échéance de deux ans. Nous nous intéressons à la zone euro, et supposons pour cela l'existence d'une politique économique commune à l'ensemble de la zone. En outre, nous cherchons à rendre compte de l'incertitude sur les résultats obtenus, en termes de fourchette. Cette incertitude, associée à tout exercice de modélisation, provient tant de la spécification des équations comportementales que des valeurs retenues pour les paramètres associés aux équations.

La démarche poursuivie dans ce papier comporte trois étapes. Dans un premier temps, nous posons un modèle DSGE en économie fermée sur la zone euro. Ce modèle contient un certain nombre de rigidités: des rigidités nominales, sur les prix et les salaires, ainsi que des rigidités réelles, matérialisées par des coûts d'ajustement sur les variations de l'investissement et sur l'utilisation du capital, et par la formation d'habitudes de consommation. Ces différentes rigidités introduisent de la persistance dans la dynamique des variables et, ainsi, permettent au modèle de mieux expliquer les données. La deuxième étape consiste à caractériser l'incertitude relative à chacun des paramètres estimés, la forme du modèle étant fixée. Pour cela, nous adoptons une approche bayésienne dans l'exercice d'estimation des paramètres: la méthode bayésienne permet en effet d'inverser la fonction de vraisemblance associée au modèle théorique retenu conditionnellement au choix des paramètres, pour construire la distribution de probabilité des paramètres conditionnellement aux données et au modèle posé. Nous effectuons enfin l'exercice de variante en simulant le modèle de façon déterministe, puisque le choc envisagé modifie son état stationnaire. L'incertitude obtenue précédemment sur les paramètres est projetée sur les résultats de la variante.

Les résultats obtenus offrent une lecture économique cohérente des effets simulés de l'annonce d'une hausse permanente du taux de TVA de la zone euro sur les variables macroéconomiques modélisées. En particulier, l'annonce de ce choc deux ans avant a pour effet de modifier immédiatement l'arbitrage intertemporel des ménages dans leurs choix : la consommation, dont ils anticipent l'augmentation du coût relatif, s'accroît progressivement entre la date de l'annonce et celle du choc. Cette hausse de la demande s'accompagne logiquement de négociations salariales à la hausse, provoquant de légères pressions inflationnistes. Parallèlement, anticipant une baisse de la production au moment du choc, conséquence d'une consommation future moindre, les agents cherchent à lisser la nécessaire adaptation de l'appareil productif. Ainsi, l'investissement se réduit dès l'annonce, la capacité des entreprises à répondre au surcroît transitoire de demande étant assurée par les variations du taux d'utilisation des capacités de production. Enfin, à l'annonce du choc, l'écart de production augmente, car les rigidités sur la fixation des salaires freinent la hausse du coût du travail pour les entreprises, qui, dans un monde parfaitement flexible, pénaliserait plus lourdement l'offre de biens et services. En outre, les simulations effectuées permettent une discussion sur l'incertitude liée à l'estimation du modèle dans les résultats. Ainsi, par exemple, l'incertitude sur la valeur des paramètres permet de construire une distribution de probabilité complète du salaire réel au moment de l'annonce du choc. Celui-ci est en effet susceptible de baisser avec une probabilité estimée à 38,6\%, si les pressions inflationnistes précèdent les hausses des salaires nominaux, plus rigides que les prix. Ainsi, nous proposons simultanément une illustration de l'intérêt des modèles DSGE pour l'analyse des comportements induits par les politiques économiques, et de la pertinence de l'estimation bayésienne pour rendre compte de l'incertitude sur les résultats obtenus. La démarche retenue pourrait être développée dans d'autres directions. Nous pourrions par exemple étendre la caractérisation 
de l'incertitude, qui porte ici uniquement sur la valeur des paramètres, à la spécification du modèle théorique retenu, en comparant les qualités d'ajustement de différents modèles, via leurs densités marginales. Il serait également intéressant de construire des prévisions macroéconomiques conditionnelles à une politique économique future. Une telle approche consisterait à effectuer un exercice similaire non pas à partir d'un état stationnaire, mais plutôt d'un état observé de l'économie

\section{Non-technical summary}

Dynamic stochastic general equilibrium (DSGE) models are based on agents' optimizing behaviour. In particular, the decisions they make take into account their expectations with regard to the future state of the economy. This property explains to some extent the advantage that several institutions in charge of economic policy find in using this kind of tools at the same time as more usual macroeconometric models. Not only they allow for realistic interpretations of macroeconomic behaviours, but they also give the possibility to simulate the effects on the economy of the announcement of a particular future economic policy.

We illustrate this possibility by studying the impact of an announced and permanent tax change. More precisely, we analyse the effect of the announcement of a 2-point increase in the effective VAT rate of the euro area two years before its implementation. We thus implicitely assume that fiscal policies are perfectly coordinated across the euro area. In addition, we try to provide a measure of the uncertainty on the simulations obtained, this uncertainty being the result of both the wrong specification of the equations and of the estimation of the parameters of the model.

In this paper, we proceed in three steps. First, we build a closed-economy DSGE model for the euro area. This model includes both nominal and real rigidities. These features give some persistence to the dynamic of the variables, and enable us to better fit data to the model. Second, we characterize the uncertainty on the values of the parameters. This is done by estimating the model with bayesian techniques: we use the likelihood function of the model given the parameters and the information we have a priori on the value of each parameter to derive the probability distribution of parameters given the set of data and the model. Third, we simulate the model deterministically, as the fiscal shock we consider affects the steady state of the model. The uncertainty on the estimated parameters is projected onto the results of the variant.

The simulations obtained are consistent with the expected effects of an announced increase in the effective VAT rate of the euro area, and are easy to interpret. In particular, the announcement immediately changes the intertemporal arbitration of households: consumption progressively increases during the two first years as they expect its relative price to grow after the measure takes place. The resulting increase in production pushes wages up, which in turn brings about slight inflationary pressures. At the same time, firms expect a marked decrease of consumption at the time the measure is implemented and they try to smooth the adaptation of their production capacities. Consequently, investment decreases immediately, which is temporarily offset by an increase in the capacity utilization rate in order for firms to be able to meet the demand. Finally, at the time of the announcement, the output gap opens, because the presence of wage rigidities slows down the increase in firms' production costs, which would have a more negative impact on the supply side of the economy in a perfectly flexible world. Besides, this exercice provides some insight into the uncertainty surrounding the results obtained. For example, computing the distribution of the parameters of the model enables us to derive the complete distribution function of the real wage in the economy at the time of the announcement: in particular, it would decrease with a probability evaluated to $38.6 \%$, which corresponds to the case where prices are less rigid than nominal wages and rise before them.

This exercise primarily aims at illustrating some advantages of DSGE models that institutions could benefit from in their analysis of the effects of economic policies on behaviours, as well as the relevance of the bayesian method for accounting for uncertainty. The approach presented in this paper could be extended in some directions: for example, uncertainty could also be characterized with regard to the choice of the model, by comparing the marginal densities of different specifications. Using the same method, we could also generate conditional forecasts, subject to future implementations of economic policies that are already announced at the time of the forecast. 


\section{Introduction}

Les modèles d'équilibre général intertemporels stochastiques (MEGIS, ou DSGE en anglais) se sont progressivement imposés comme des outils de la modélisation macroéconomique. Initialement développés dans le monde académique, leur utilisation s'est plus récemment étendue aux institutions en charge de la politique économique, suite aux travaux de Smets et Wouters (2003). Ces modèles présentent des avantages par rapport aux modèles macroéconométriques utilisés habituellement dans ces institutions ${ }^{1}$, parmis lesquels deux nous paraissent majeurs. D'une part, leur construction repose sur un cadre théorique cohérent fondé sur des comportements optimisateurs des agents, ce qui n'est pas le cas des modèles économétriques. D'autre part, tournés vers le passé, ces modèles macroéconométriques ne permettent pas de tenir compte de l'impact des anticipations des agents sur l'économie, et sont la cible de la critique de Lucas $(1976)^{2}$. Néanmoins, les modèles d'équilibre général restent beaucoup trop stylisés pour pouvoir s'adapter au cadre comptable désagrégé généralement exploité dans le discours institutionnel.

Comme la prise en compte des anticipations est indispensable dans la modélisation de variantes relatives à des chocs futurs annoncés, nous utilisons dans cet article un modèle d'équilibre général intertemporel pour analyser les effets d'une modification permanente et annoncée d'une politique fiscale future. À titre d'illustration, nous nous intéressons aux effets d'une politique consistant à augmenter le taux de TVA dans la zone euro ceteris paribus.

Comme nous ne connaissons pas de façon certaine le modèle qui génère les données (DGP, pour data generating process), il convient de rendre compte de l'incertitude sur les résultats obtenus, en raisonnant en termes de fourchette. L'incertitude sur le DGP peut porter sur la spécification d'un modèle paramétré et sur la valeur de ses paramètres. Dans la suite, nous allons fixer la forme du modèle, puis nous caractériserons l'incertitude sur le DGP à l'aide d'une densité jointe sur les paramètres du modèle. Nous projetterons alors cette incertitude dans l'espace des variantes.

La démarche poursuivie dans ce papier procède en trois étapes. Dans un premier temps, nous posons un modèle DSGE en économie fermée sur la zone euro. Nous suivons Smets et Wouters (2003); le modèle contient un certain nombre de rigidités nominales, sur les prix et les salaires, ainsi que réelles, avec coût d'ajustement sur l'investissement et l'utilisation du capital. La deuxième étape consiste à caractériser l'incertitude relative au modèle en construisant la densité jointe de ses paramètres ; c'est pourquoi nous adoptons une approche bayésienne. Le modèle définit la densité jointe d'un ensemble de variables (in-

1. Voir, par exemple, le modèle MASCOTTE, Baghli et al. (2004).

2. Voir Fève (2005) pour une discussion des différentes méthodologies. 
flation, salaire réel, etc.) conditionnellement aux paramètres; la méthode bayésienne permet d'inverser celle-ci et de mettre à jour nos croyances a priori sur le DGP pour construire la densité jointe des paramètres conditionnellement aux données (la densité a posteriori). Dans la dernière étape, on traduit la densité postérieure des paramètres en une incertitude sur les variantes.

L'exercice de variante est basé sur une version déterministe du modèle DSGE (ie les variances des chocs stochastiques sont nulles). Le modèle DSGE n'est indispensable que dans la deuxième étape, pour écrire la fonction de vraisemblance associée au modèle théorique et caractériser l'incertitude sur les paramètres structurels. Le choix du modèle est évidemment discutable. Nous prenons le parti de rester le plus proche possible du modèle considéré par Smets et Wouters (2003). Ce choix est motivé par le relativement bon comportement du modèle lorsqu'il est confronté aux données ${ }^{3}$ et par le fait qu'il se soit imposé dans le monde instutitionnel comme un modèle cannonique.

La suite de l'article se présente de la manière suivante ; la section 2 décrit les équations du modèle, puis nous présentons les résultats de l'estimation dans la section 3. Un exercice de variante est discuté dans la section 4. La section 5 conclut.

\section{Description du modèle}

Le modèle choisi dans ce papier considère la zone euro comme une économie fermée. Par de nombreux aspects, il se rapproche du modèle développé par Smets et Wouters (2003): rigidités nominales à la Calvo (1983) sur la formation des prix et des salaires, rigidités réelles telles que des coûts d'ajustement sur le capital et sur le niveau d'utilisation des capacités de production, ainsi que des formations d'habitudes sur la consommation. De plus, nous ajoutons des chocs fiscaux au modèle original afin de tenir compte des effets de modifications des prélèvements obligatoires.

\subsection{Les ménages}

Nous considérons un continuum de ménages $m \in[0,1]$, chacun offrant un travail différencié. L'utilité instantanée de la consommation de chaque ménage dépend positivement de la consommation $C_{t}^{m}$ relativement à une variable d'habitude externe $H_{t}^{m}: U_{t}^{m}=\left(C_{t}^{m}-H_{t}^{m}\right)^{1-\sigma_{c}} /\left(1-\sigma_{c}\right)$, où $\sigma_{c}$ correspond à l'élasticité intertemporelle de substitution de la consommation. On suppose que la variable d'habitude externe est proportionnelle à la consommation agrégée passée: $H_{t}^{m}=h C_{t-1}^{m}$. La désutilité instantanée du travail de chaque ménage dépend positivement du travail $l_{t}^{m}: V_{t}^{m}=\varepsilon_{t}^{L}\left(l_{t}^{m}\right)^{1+\sigma_{l}} /\left(1+\sigma_{l}\right)$

3. Par exemple, les deux auteurs montrent que la qualité d'ajustement de ce modèle est comparable à celle d'un modèle VAR (ie un modèle qui n'exploite aucune contrainte théorique). 
où $\sigma_{l}$ correspond à l'élasticité de l'effort de travail et $\varepsilon_{t}^{L}$ représente un choc d'offre de travail dont le logarithme suit un processus $\mathrm{AR}(1)$. Chaque ménage $m$ maximise une fonction d'utilité intertemporelle $\mathcal{U}_{t}^{m}=\mathbb{E}_{t} \sum_{j=0}^{\infty} \beta^{j} \varepsilon_{t+j}^{B}\left(U_{t+j}^{m}-V_{t+j}^{m}\right)$, avec $\varepsilon_{t}^{B}$ un choc de préférence dont le logarithme suit un AR(1) et $\beta$ le facteur d'escompte social.

Le revenu total des ménages est la somme des revenus salariaux augmentés des flux nets issus de la détention de titres contingents ${ }^{4}\left(A_{t}^{m}\right)$, des revenus du capital détenu diminués du coût $\psi\left(z_{t}\right)$ lié aux variations du taux d'utilisation des capacités de production ${ }^{5}\left(z_{t}\right)$, des dividendes versés par les firmes du secteur intermédiaire en concurrence imparfaite, et des transferts nets du gouvernement $\Omega_{t}$. De plus, le revenu des ménages est soumis à deux taxes, portant sur les revenus du travail $\left(\tau_{t}^{W}\right)$ et du capital $\left(\tau_{t}^{K}\right)^{6}$. Les revenus du ménage $m$ s'écrivent alors:

$$
Y_{t}=\left(\tau_{t}^{W} w_{t}^{m} l_{t}^{m}+A_{t}^{m}\right)+\left(\tau_{t}^{K} r_{t}^{K} z_{t} K_{t-1}-\psi\left(z_{t}\right) K_{t-1}\right)+D i v_{t}+\Omega_{t}
$$

Les ménages maximisent leur fonction objectif sous la contrainte budgétaire intertemporelle donnée par: $B_{t} /\left(R_{t} P_{t}\right) \leq B_{t-1} / P_{t}+Y_{t}-\tau_{t}^{C} C_{t}-I_{t}$. Ils détiennent leur richesse sous forme de titres $B_{t}$ et de capital. Le revenu et la richesse des ménages peuvent être utilisés pour la consommation et l'investissement en capital physique, dont la loi d'évolution s'écrit:

$$
K_{t}=(1-\delta) K_{t-1}+\left[1-S\left(\varepsilon_{t}^{I} \frac{I_{t}}{I_{t-1}}\right)\right] I_{t}
$$

où $\varepsilon_{t}^{I}$ est un choc déformant le coût d'ajustement $S(\cdot)$, fonction qui vérifie $S(1)=S^{\prime}(1)=0$, et $\delta \in] 0,1[$ le taux de dépréciation. Les titres sont détenus sur une période et sont rémunérés au facteur d'intérêt nominal $R_{t}$. Par ailleurs, un facteur de taxe $\tau_{t}^{C}$ est appliqué à la consommation.

\subsubsection{Comportements d'épargne et de consommation}

La maximisation de la fonction objectif des ménages sous la contrainte budgétaire par rapport à la consommation et la détention d'actifs fournit les conditions d'optimalité suivantes:

$$
\mathbb{E}_{t}\left\{\beta R_{t} \frac{\lambda_{t+1} P_{t}}{\lambda_{t} P_{t+1}}\right\}=1
$$

où $\lambda_{t}$ correspond à l'utilité marginale de la consommation, donnée par:

$$
\lambda_{t}=\frac{\varepsilon_{t}^{B}}{\tau_{t}^{C}}\left(C_{t}-H_{t}\right)^{-\sigma_{c}}
$$

4. L'hypothèse de la détention de titres contingents implique que les ménages sont assurés contre les variations de leur revenus différentiés du travail de telle sorte que les choix intertemporels des ménages sont identiques, tout en gardant des salaires différenciés (Christiano et al., 2005).

5. On suppose qu'à l'état stationnaire, la fonction $\psi(\cdot)$ vérifie $\psi(\bar{z})=0$, où $\bar{z}$ est la valeur à l'équilibre du taux d'utilisation des capacités de production. De plus, on suppose $\psi^{\prime \prime}(\bar{z}) \neq 0$ et on pose $\Psi=\psi^{\prime}(\bar{z}) / \psi^{\prime \prime}(\bar{z})$.

6. Les deux variables $\tau_{t}^{W}$ et $\tau_{t}^{K}$ sont inférieures à un et représentent les parts disponibles des revenus salarial et financier (en dehors du coût lié aux variations du taux d'utilisation du capital). 
La combinaison de ces deux équations donne la condition de premier ordre usuelle pour la croissance de la consommation, tenant compte de l'existence de la formation d'habitudes externes. En notant $\pi_{t}=$ $P_{t} / P_{t-1}$, l'arbitrage intertemporel est résumé par:

$$
\frac{\varepsilon_{t}^{B}}{\tau_{t}^{C}}\left(C_{t}-h C_{t-1}\right)^{-\sigma_{c}}=\mathbb{E}_{t}\left\{\beta \frac{\varepsilon_{t+1}^{B}}{\tau_{t+1}^{C}}\left(C_{t+1}-h C_{t}\right)^{-\sigma_{c}} \frac{R_{t}}{\pi_{t+1}}\right\}
$$

\subsubsection{Investissement et accumulation du capital}

Les ménages détiennent le stock de capital qu'ils louent aux firmes du secteur intermédiaire au taux $r_{t}^{K}$. Une augmentation de l'offre de services de capital peut provenir soit de l'investissement, utilisable la période suivante, soit de l'augmentation du taux d'utilisation du capital déjà installé; chacune de ces deux opérations génère un coût, pris en compte par les fonctions $S(\cdot)$ et $\psi(\cdot)$.

En notant le prix relatif du capital $Q_{t}=\mu_{t} / \lambda_{t}$, où $\mu_{t}$ est le prix implicite d'une unité de capital, les conditions d'optimalité par rapport au choix sur le niveau de capital, de l'investissement et du taux d'utilisation des capacités donnent les relations suivantes:

$$
\begin{gathered}
Q_{t}=\mathbb{E}_{t}\left\{\beta \frac{\lambda_{t+1}}{\lambda_{t}}\left[\tau_{t+1}^{K} r_{t+1}^{K} z_{t+1}-\psi\left(z_{t+1}\right)+Q_{t+1}(1-\delta)\right]\right\} \\
Q_{t}\left[\left(1-S\left(\frac{\varepsilon_{t}^{I} I_{t}}{I_{t-1}}\right)-\frac{\varepsilon_{t}^{I} I_{t}}{I_{t-1}} S^{\prime}\left(\frac{\varepsilon_{t}^{I} I_{t}}{I_{t-1}}\right)\right)\right]+\mathbb{E}_{t}\left\{\beta Q_{t+1} \frac{\lambda_{t+1}}{\lambda_{t}} \varepsilon_{t+1}^{I}\left(\frac{I_{t+1}}{I_{t}}\right)^{2} S^{\prime}\left(\varepsilon_{t+1}^{I} \frac{I_{t+1}}{I_{t}}\right)\right\}=1 \\
\tau_{t}^{K} r_{t}^{K}=\psi^{\prime}\left(z_{t}\right)
\end{gathered}
$$

\subsubsection{Offre de travail}

Les ménages offrant un travail différencié, ils conservent un pouvoir de marché sur la détermination de leur salaire. Nous supposons l'existence d'une agence qui propose une offre de travail agrégée $L_{t}$, obtenue par la combinaison de l'offre de travail de chaque ménage selon une fonction de type DixitStiglitz: $L_{t}=\left(\int_{0}^{1}\left(l_{t}^{m}\right)^{\frac{\nu-1}{\nu}} \mathrm{d} m\right)^{\frac{\nu}{\nu-1}}$ où $\nu$ se définit comme l'elasticité de l'effort de travail au salaire. L'agence d'emploi maximise son profit, étant donnés les salaires nominaux différenciés des ménages $P_{t} w_{t}^{m}$ et l'offre de salaire nominal agrégée $P_{t} w_{t}{ }^{7}$. Il en résulte la fonction de demande suivante :

$$
l_{t}^{m}=\left(\frac{w_{t}^{m}}{w_{t}}\right)^{-\nu} L_{t}
$$

7. On note en minuscule le salaire réel et en majuscule le salaire nominal. 
En utilisant la condition de profit nul à l'optimum, nous obtenons la définition du salaire nominal agrégé : $W_{t}=\left(\int_{0}^{1}\left(W_{t}^{m}\right)^{1-\nu} \mathrm{d} m\right)^{\frac{1}{1-\nu}}$. Néanmoins, comme Ecerg et al. (2000), nous supposons que les ménages ne peuvent pas optimiser leur salaire à chaque date. Avec la probabilité $\xi_{w}$, le ménage $m$ ne peut pas ajuster son salaire de manière optimale. Le salaire nominal suit alors l'évolution suivante: $W_{t}^{m}=\bar{\pi}_{t}^{\gamma_{w}} \pi_{t-1}^{1-\gamma_{w}} W_{t-1}^{m}$, ie la variation du salaire instantanée d'un ménage qui n'a pas eu l'opportunité de choisir le niveau optimal est indexée sur une combinaison convexe de l'inflation passée $\pi_{t-1}$ et de la cible d'inflation, éventuellement variable, de la banque centrale $\bar{\pi}_{t}$. Avec la probabilité $1-\xi_{w}$, le ménage $m$ a la possibilité de choisir son niveau optimal de salaire $\tilde{W}_{t}^{m}$.

Les conditions d'optimalité issues de la maximisation de la fonction objectif du ménage $m$ par rapport au choix du salaire optimal, en tenant compte des chocs idiosyncratiques qu'il subit, donnent les relations suivantes:

$$
\pi_{t} \tilde{w}_{t}^{m}=\frac{\nu}{1-\nu} \frac{\mathcal{H}_{1, t}}{\mathcal{H}_{2, t}}
$$

avec $\mathcal{H}_{1, t}$ et $\mathcal{H}_{2, t}$, deux fonctions vérifiant les récurrences:

$$
\begin{aligned}
\mathcal{H}_{1, t} & =\varepsilon_{t}^{B} V_{t}^{m^{\prime}} l_{t}^{m}+\beta \xi_{w} \mathbb{E}_{t}\left\{\mathcal{H}_{1, t+1}\right\} \\
\mathcal{H}_{2, t} & =\frac{\varepsilon_{t}^{B} U_{t}^{m^{\prime}} \tau_{t}^{W} l_{t}^{m}}{\tau_{t}^{C} \pi_{t}}+\beta \xi_{w} \bar{\pi}_{t+1}^{\gamma_{w}} \pi_{t}^{-\gamma_{w}} \mathbb{E}_{t}\left\{\mathcal{H}_{2, t+1}\right\}
\end{aligned}
$$

$U_{t}^{m^{\prime}}$ et $V^{m^{\prime}}$ étant respectivement l'utilité marginale de la consommation et la désultilité marginale du travail, définis par:

$$
\begin{aligned}
V_{t}^{m^{\prime}} & =\varepsilon_{t}^{L}\left(l_{t}^{m}\right)^{\sigma_{l}} \\
U_{t}^{m^{\prime}} & =\left(C_{t}-h C_{t-1}\right)^{-\sigma_{c}}
\end{aligned}
$$

Enfin, la distinction entre les ménages qui ont la possibilité d'optimiser leur salaire et ceux qui ne l'ont pas amène à réécrire la définition du salaire agrégé:

$$
w_{t}^{1-\nu}=\left(1-\xi_{w}\right) \tilde{w}_{t}^{1-\nu}+\xi_{w}\left(\frac{\bar{\pi}_{t}^{\gamma_{w}} \pi_{t-1}^{1-\gamma_{w}}}{\pi_{t}} w_{t-1}\right)^{1-\nu}
$$

\subsection{Les firmes et la fixation des prix}

\subsubsection{Secteur du bien final}

Le secteur du bien final est caractérisé par une firme représentative qui agrège la production d'un continuum de firmes intermédiaires $f \in[0,1]$. Ces firmes produisent chacune un bien différencié $y_{t}^{f}$ et sont en concurrence monopolistique. La fonction d'agrégation de type Dixit-Stiglitz est définie par: $Y_{t}=\left(\int_{0}^{1}\left(y_{t}^{f}\right)^{\frac{\epsilon-1}{\epsilon}} \mathrm{d} f\right)^{\frac{\epsilon}{\epsilon-1}}$ où $\epsilon$ est l'élasticité prix de la demande. Cette firme représentative maximise 
son profit étant donné le prix des biens intermédiaires $P_{t}^{f}$ et le prix du bien final $P_{t}$. Il résulte de ce comportement les fonctions de demande suivantes pour les biens intermédiaires : $y_{t}^{f}=\left(P_{t}^{f} / P_{t}\right)^{-\epsilon} Y_{t}$. La définition du prix du bien final s'obtient alors en utilisant la condition de profit nul à l'optimum et donne : $P_{t}=\left(\int_{0}^{1}\left(P_{t}^{f}\right)^{1-\epsilon} \mathrm{d} f\right)^{\frac{1}{1-\epsilon}}$.

\subsubsection{Secteur des biens intermédiaires}

Nous supposons que la technologie de production de toutes les firmes du secteur des biens intermédiaires est identique et peut être représentee par une fonction de type Cobb-Douglas: $y_{t}^{f}=\varepsilon_{t}^{a}\left(\widetilde{K}_{t}^{f}\right)^{\alpha}\left(L_{t}^{f}\right)^{1-\alpha}-$ $\Phi$ où $\tilde{K}_{t}^{f}$ est le capital utilisé défini par:

$$
\tilde{K}_{t}^{f}=z_{t} K_{t}^{f}
$$

$\varepsilon_{t}^{a}$ est un choc de productivité, et $\Phi>0$ représente un coût fixe tel que le profit d'une firme intermédiaire est nul dans le long terme ${ }^{8}$.

En supposant que les marchés de facteurs de production sont parfaitement concurrentiels, une firme $f \in$ $[0,1]$ cherche à minimiser ses coûts sous sa contrainte technologique. La résolution de son programme donne la relation suivante :

$$
\frac{\tau_{t}^{l} w_{t} L_{t}^{f}}{\tau_{t}^{r} r_{t}^{K} \widetilde{K}_{t}^{f}}=\frac{1-\alpha}{\alpha}, \forall f \in[0,1]
$$

où $\tau_{t}^{i}, i \in\{l, r\}$, sont deux facteurs de taxes sur les coûts du travail et du capital. Les décisions sur la combinaison optimale des facteurs de production sont alors identiques entre les firmes intermédiaires. Après réarrangement de ces équations, on peut donner l'expression du coût marginal réel des firmes intermédiaires:

$$
m c_{t}=\frac{\left(\tau_{t}^{r} r_{t}^{K}\right)^{\alpha}\left(\tau_{t}^{l} w_{t}\right)^{1-\alpha}}{\varepsilon_{t}^{a} \alpha^{\alpha}(1-\alpha)^{1-\alpha}}
$$

Le coût marginal réel, qui apparaîtra dans la courbe de Phillips, augmente avec les impôts et diminue avec un choc de productivité. On peut noter aussi qu'il ne dépend pas de $f$.

Le profit nominal de la firme $f$ à la date $t$ s'écrit: $\Pi_{t}^{f}=\left(\tau_{t}^{y} P_{t}^{f}-P_{t} m c_{t}\right)\left(P_{t}^{f} / P_{t}\right)^{-\epsilon} Y_{t}-P_{t} m c_{t} \Phi$, où $\tau_{t}^{y}$ est un choc fiscal sur le revenu de la firme qui affecte son taux de marge. Les firmes n'ont pas la possibilité de fixer leur prix de manière optimale à chaque date. Avec la probabilité $\xi_{p}$, la firme $f$ ne peut pas réoptimiser son prix; celui ci suit alors la règle d'évolution : $P_{t}^{f}=\bar{\pi}_{t}^{\gamma_{p}} \pi_{t-1}^{1-\gamma_{p}} P_{t-1}^{f}$, i.e le prix d'une firme qui n'a pas l'opportunité de le fixer à son niveau optimal, est le résultat d'une combinaison convexe entre l'inflation totale passée et la cible d'inflation de la banque centrale. Le temps moyen pendant lequel une firme ne peut pas optimiser son prix est $1 /\left(1-\xi_{p}\right)$. Avec la probabilité $1-\xi_{p}$, la

8. Ce coût fixe s'écrit alors $\Phi=\frac{\bar{Y}}{\epsilon-1}$, où $\bar{Y}$ est la valeur de la production à l'état stationnaire. 
firme $f$ peut choisir le prix optimal $\tilde{P}_{t}^{f}$. Posons $p_{t} \equiv \tilde{P}_{t}^{f} / P_{t}$ le prix relatif de la firme $f$. Le programme d'optimisation des firmes intermédiaires étant tourné vers le futur, le prix relatif ne dépend pas de $f$. Les conditions du premier ordre définissent alors le système suivant:

$$
\frac{\pi_{t}}{\bar{\pi}_{t}^{\gamma_{p}} \pi_{t-1}^{1-\gamma_{p}}} p_{t}=\frac{\epsilon}{\epsilon-1} \frac{\mathcal{Q}_{1, t}}{\mathcal{Q}_{2, t}}
$$

avec

$$
\begin{aligned}
\mathcal{Q}_{1, t} & =\pi_{t}^{\epsilon} \bar{\pi}_{t}^{-\epsilon \gamma_{p}} \pi_{t-1}^{-\left(1-\gamma_{p}\right) \epsilon} \lambda_{t} Y_{t} m c_{t}+\beta \xi_{p} \pi_{t}^{\epsilon} \pi_{t-1}^{-\left(1-\gamma_{p}\right) \epsilon} \bar{\pi}_{t}^{-\gamma_{p} \epsilon} \mathbb{E}_{t}\left[\mathcal{Q}_{1, t+1}\right] \\
\mathcal{Q}_{2, t} & =\pi_{t}^{\epsilon-1} \bar{\pi}_{t}^{\gamma_{p}(1-\epsilon)} \pi_{t-1}^{\left(1-\gamma_{p}\right)(1-\epsilon)} \lambda_{t} \tau_{t}^{y} Y_{t}+\beta \xi_{p} \pi_{t}^{\epsilon-1} \pi_{t-1}^{\left(1-\gamma_{p}\right)(1-\epsilon)} \bar{\pi}_{t}^{\gamma_{p}(1-\epsilon)} \mathbb{E}_{t}\left[\mathcal{Q}_{2, t+1}\right]
\end{aligned}
$$

Pour compléter cette analyse, nous tenons compte dans l'expression du prix agrégé de l'hétérogénéité des firmes intermédiaires, ce qui donne:

$$
\left\{\left(1-\xi_{p}\right) p_{t}^{1-\epsilon}+\xi_{p}\left[\bar{\pi}_{t}^{\gamma_{p}} \frac{\pi_{t-1}^{1-\gamma_{p}}}{\pi_{t}}\right]^{1-\epsilon}\right\}^{\frac{1}{1-\epsilon}}=1
$$

Les équations (15) et (16)-(19) décrivent complétement la dynamique des prix dans ce modèle, étant donnés les salaires et taux d'intérêt réels.

\subsubsection{Distorsion de prix et agrégation}

Nous devons vérifier que malgré l'hétérogénéité des prix et salaires induite par les rigidités à la Calvo (1983), nous sommes capables de définir une économie agrégée. À partir de la frontière des prix de facteurs, nous savons que le rapport entre facteurs de production doit être constant entre les firmes $f \in[0,1]$. En conséquence, en définissant $\widetilde{K}(t)=\int_{0}^{1} \widetilde{K}_{t}^{f} \mathrm{~d} f$ et $L(t)=\int_{0}^{1} L_{t}^{f} \mathrm{~d} f$ le stock de capital utilisé agrégé et le travail agrégé, nous avons :

$$
\frac{\widetilde{K}_{t}}{L_{t}}=\frac{\alpha}{1-\alpha} \frac{\tau_{t}^{l} w_{t}}{\tau_{t}^{r} r_{t}^{K}}=\frac{\widetilde{K}_{t}^{f}}{L_{t}^{f}}
$$

En exprimant $L_{t}^{f}$ comme une fonction de $\widetilde{K}_{t}^{f}$ dans la définition de la technologie des firmes intermédiaires, nous obtenons : $y_{t}^{f}=\varepsilon_{t}^{a}\left(L_{t} / \widetilde{K}_{t}\right)^{1-\alpha} \widetilde{K}_{t}^{f}-\Phi$. En intégrant cette expression par rapport à $f$ sur $[0,1]$, nous obtenons une expression pour la production agrégée : $y_{t}=\int_{0}^{1} y_{t}^{f} \mathrm{~d} f=\varepsilon_{t}^{a} \widetilde{K}_{t}^{\alpha} L_{t}^{1-\alpha}-\Phi$ qui est a priori différente de $Y_{t}$ définie comme un agrégat Dixit-Stiglitz, impliquant une élasticité de substitution entre les biens intermédiaires. À l'aide des équations de demande adressée à chaque firme intermédiaire, nous avons : $\int_{0}^{1} y_{t}^{f} \mathrm{~d} f=Y_{t} \int_{0}^{1}\left(P_{t}^{f} / P_{t}\right)^{-\epsilon} \mathrm{d} f=D_{p, t} Y_{t}$, où $D_{p, t}=\int_{0}^{1}\left(P_{t}^{f} / P_{t}\right)^{-\epsilon} \mathrm{d} f$ mesure la distorsion de prix telle que:

$$
D_{p, t} Y_{t}=\varepsilon_{t}^{a} \widetilde{K}_{t}^{\alpha} L_{t}^{1-\alpha}-\Phi
$$


Il est nécessaire d'obtenir une forme récursive de cette distorsion afin de simuler le modèle non linéaire ${ }^{9}$. Nous avons donc à partir de la définition de $D_{p, t}$ :

$$
D_{p, t}=\left(1-\xi_{p}\right) p_{t}^{-\epsilon}+\xi_{p}\left(\frac{\bar{\pi}_{t}^{\gamma_{p}} \pi_{t-1}^{1-\gamma_{p}}}{\pi_{t-1}}\right)^{-\epsilon} D_{p, t-1}
$$

À l'état stationnaire déterministe, nous avons $D_{p, t}=1$, ie il n'y a plus de distorsion de prix dans le long terme.

\section{3 Équilibre ressource-emploi et bouclage du modèle}

Le budget de l'État étant équilibré à chaque date par les transferts aux ménages, $\Omega_{t}$, la production est égale à la demande en consommation et investissement, augmentée du coût d'ajustement du capital et des dépenses publiques :

$$
Y_{t}=C_{t}+I_{t}+\psi\left(z_{t}\right) K_{t-1}+G_{t}
$$

où les dépenses publiques $G_{t}$ suivent un processus $\operatorname{AR}(1)$ :

$$
\log G_{t}=\left(1-\rho_{G}\right) \log \bar{G}+\rho_{G} \log G_{t-1}+\nu_{t}^{G}, \nu_{t}^{G} \sim \mathcal{N}\left(0, \sigma_{G}\right)
$$

Pour boucler le modèle nous considérons une régle de Taylor. L'autorité monétaire détermine une cible d'inflation ${ }^{10}$

$$
\log \bar{\pi}_{t}=\rho_{\pi} \log \bar{\pi}_{t-1}+\left(1-\rho_{\pi}\right) \log \bar{\pi}+\nu_{t}^{\pi}
$$

puis contôle le taux d'intérêt nominal en réagissant (i) aux déviations de l'inflation à sa cible, (ii) à l'écart de production défini comme la différence entre la production effective et celle obtenue en absence de rigidités nominales $Y_{t}^{*}$. Ainsi, la règle de Taylor s'écrit :

$$
R_{t}=\bar{R}^{1-\rho} R_{t-1}^{\rho}\left[\bar{\pi}_{t}\left(\frac{\pi_{t-1}}{\bar{\pi}_{t}}\right)^{r_{\pi}}\left(\frac{Y_{t}}{Y_{t}^{*}}\right)^{r_{Y}}\right]^{1-\rho}\left(\frac{\pi_{t}}{\pi_{t-1}}\right)^{r_{\Delta \pi}}\left(\frac{Y_{t} Y_{t-1}^{*}}{Y_{t-1} Y_{t}^{*}}\right)^{r_{\Delta y}} e^{\eta_{t}^{R}}
$$

La présence du retard $R_{t-1}$ traduit la volonté de l'autorité monétaire de lisser la dynamique du taux d'intérêt nominal. Pour évaluer $Y_{t}^{*}$, nous augmentons notre économie avec un nouveau modèle dans lequel il n'y a pas de rigidités nominales (ie on pose $\xi_{p}=\xi_{w}=0$ ).

\section{Incertitude sur le modèle}

Dans la section précédente, nous avons posé un modèle paramétré comme processus générateur des données (DGP); on note $\theta \in \Theta \subseteq \mathbb{R}^{n}$ le vecteur des paramètres structurels. Des valeurs différentes de $\theta$ définissent différents DGP. Ainsi, une incertitude sur $\theta$, caractérisée par une densité de probabilité,

9. Avec une approximation de Taylor à l'ordre 1 autour de l'état stationnaire déterministe, cette distorsion disparait.

10. Le paramètre $\bar{\pi}$ représente le facteur d'inflation cible à long terme. 
définit un continuum de modèles possibles. L'estimation bayésienne de $\theta$, en confrontant nos a priori aux données, permet de réviser l'ensemble des modèles possibles. L'estimation est effectuée sur une forme simplifiée du modèle, dans laquelle les chocs fiscaux ont été neutralisés (les facteurs de taxes sont alors fixés à leurs valeurs à l'état stationnaire).

\subsection{Les croyances a priori}

Nos croyances sont dégénérées dans certaines directions : pour certains paramètres nous n'avons aucune incertitude, ils sont étalonnés. Notre incertitude ne porte que sur la spécification des chocs, sur les paramètres qui définissent les rigidités réelles et nominales et sur les paramètres qui définissent les préférences des ménages ${ }^{11}$.

Un certain nombre de paramètres sont directement calibrés à partir des données (voir la section 3.2). On obtient ainsi pour le taux d'intérêt nominal annuel $\bar{R}=4,51 \%$ et le taux d'inflation annuel $\bar{\pi}=2,34 \%$, ce qui implique un facteur d'escompte $\beta$ égal à 0,994. Par ailleurs, le taux d'utilisation des capacités de production à l'état stationnaire est calibré à $\bar{z}=0,82$. Enfin, la part des dépenses publiques dans le PIB est calibrée à 24,1\%. Les paramètres decrivant la fiscalité ont été calibrés à partir des mêmes données. Nous obtenons ainsi un facteur de TVA $\tau^{C}=1.13$, des taux de charges salariales et charges patronales égaux respectivement à $13 \%$ et $30 \%$, ce qui implique $\tau^{W}=0.87$ et $\tau^{l}=1.30$. La fiscalité sur les revenus du capital, le profit des firmes et la location du capital ont été neutralisés, en retenant $\tau^{K}=\tau^{Y}=\tau^{r}=1$.

Concernant les paramètres non directement calibrés à partir des données, on retient un taux de dépréciation du capital $\delta$ égal à 0,025 , soit un taux annuel de $10 \%$. Le paramètre $\alpha$ est calibré à 0,30 , ce qui correspond à la part du capital dans la valeur ajoutée. Pour finir, les élasticités de substitution du travail et des biens intermédiaires dans la production sont calibrés respectivement à $\lambda_{w}=\frac{1}{\nu-1}=0,5$ et $\lambda_{p}=\frac{1}{\epsilon-1}=0,3$.

Nous retenons comme fonction de coût d'ajustement sur le niveau de l'investissement $S(x)=\frac{1}{2 \varphi}(1-$ $x)^{2}$, qui vérifie les contraintes $S(1)=S^{\prime}(1)=0$. La fonction de coût lié aux variations du taux d'utilisation des capacités de production $\psi$ doit vérifier, à l'équilibre, $\psi(\bar{z})=0$; on retiendra la forme

11. Le partage entre les paramètres certains (étalonnés) et incertains (estimés) mérite discussion. Nous suivons ici l'usage en n'estimant pas les paramètres dont nous savons à l'avance qu'il est difficile de les identifier avec des données filtrées. Par exemple, il est difficile d'identifier le facteur d'escompte $\beta$ si les données (filtrées) n'apportent pas d'information sur le niveau moyen du taux d'intérêt réel, ce paramètre sera donc étalonné. Etant donné notre problématique, il serait plus pertinent de n'étalonner aucun paramètre en associant une densité a priori à chaque paramètre. Si ces paramètres, à l'instar de $\beta$, ne sont pas (ou peu) identifiable la densité postérieure sera identique (ou proche) à la densité a priori. Autrement dit, la confrontation aux donnés ne réduit pas notre incertitude sur ces paramètres. En plus d'augmenter la dimension du problème, on comprendra plus loin que cela n'affecterait vraisemblablement que marginalement les résultats sur nos variantes, dans la mesure où on ne considère pas l'incertitude sur les paramètres qui affectent l'état stationnaire. C'est pourquoi nous suivons l'usage en étalonnant une partie des paramètres. 
$\psi(z)=\exp \left(\frac{z-\bar{z}}{\Psi}\right)-1$

Les densités a priori associées aux paramètres pour lesquels nous sommes incertains sont présentées dans les premières colonnes du tableau 1. Les écart-types relatifs aux chocs sont estimés sur la base de priors non informatifs (distributions uniformes). L'incertitude sur l'élasticité de substitution intertemporelle de la consommation, $\sigma_{c}$, et l'élastictité de l'offre de travail, $\sigma_{l}$, sont respectivement caractérisées par des lois normales $\mathcal{N}(1,5 ; 0,5)$ et $\mathcal{N}(3,5 ; 0,5)$. Concernant les paramètres relatifs aux rigidités nominales, nous avons utilisé des distributions a priori informatives ; les densités a priori sur les probabilités de Calvo, $\xi_{p}$ et $\xi_{w}$, sont des beta $\mathcal{B}(0,75 ; 0,05)^{12}$. Dans le cas de données trimestrielles, la valeur de 0,75 correspond à une réévaluation des salaires ou des prix une fois par an. Les paramètres d'indexation sont des beta $\mathcal{B}(0,25 ; 0,15)$. L'incertitude a priori sur ces paramètres est plus grande que l'incertitude sur les probabilités de Calvo. Notre règle de Taylor a priori est conforme aux règles généralement envisagées dans la littérature (voir par exemple Smets et Wouters, 2003). L'incertitude sur les paramètres autorégressifs est spécifiée à l'aide de distributions beta ou uniforme.

\subsection{Données}

L'estimation des paramètres a été effectuée à partir de données trimestrielles de la zone euro. Les données retenues sont celles utilisées par le modèle Amazone développé par la Banque de France. Elles sont issues des comptes nationaux d'Eurostat, à l'exception de la série de TUC qui est fournie par la BRI, et du taux d'intérêt qui est le taux Euribor à 3 mois. Le facteur d'inflation est défini comme le rapport des déflateurs du PIB sur deux périodes consécutives. Les paramètres du modèle sont identifiés à l'aide de sept variables : le PIB en volume, la consommation des ménages en volume, l'investissement en volume, le taux d'intérêt, le TUC, le salaire par tête et un facteur d'inflation, observées entre 1991Q2 et 2005Q4. À la différence de Smets et Wouters (2003), nous n'utilisons pas l'emploi comme une proxy des heures travaillées. Les données sont corrigées de leur tendance, supposée log-linéaire, et centrées.

\subsection{Les croyances a posteriori}

Afin de pouvoir estimer le modèle avec les sept variables observables, la structure stochastique du modèle comprend sept chocs ${ }^{13}$, dont cinq chocs autorégressifs :

$$
\begin{gathered}
\log \varepsilon_{t}^{j}=\zeta^{j} \log \varepsilon_{t-1}^{j}+\eta_{t}^{j}, \eta_{t}^{j} \sim \mathcal{N}\left(0, \sigma_{j}\right) \quad \text { pour } j=a, B, L \\
\log G_{t}=\left(1-\rho_{G}\right) \log \bar{G}+\rho_{G} \log G_{t-1}+\nu_{t}^{G}, \nu_{t}^{G} \sim \mathcal{N}\left(0, \sigma_{G}\right) \\
\log \bar{\pi}_{t}=\rho_{\pi} \log \bar{\pi}_{t-1}+\left(1-\rho_{\pi}\right) \log \bar{\pi}+\nu_{t}^{\pi}, \nu_{t}^{\pi} \sim \mathcal{N}\left(0, \sigma_{\pi}\right)
\end{gathered}
$$

12. Contrairement à l'usage, nous définissons ici la distribution beta par l'espérance et l'écart-type.

13. Pour écrire la vraisemblance du modèle il faut qu'il y ait au moins autant de sources d'aléa que de variables observables. 


\begin{tabular}{|c|c|c|c|c|c|c|c|}
\hline & \multicolumn{3}{|c|}{ Croyances a priori } & \multicolumn{4}{|c|}{ Croyances a posteriori } \\
\hline & Distribution & Espérance & Écart-type & Espérance & Écart-type & $\mathcal{I}_{1}$ & $\mathcal{I}_{2}$ \\
\hline$\sigma_{c}$ & normale & 1.500 & 0.5000 & 1.989 & 0.4116 & 1.3118 & 2.6692 \\
\hline$\sigma_{l}$ & normale & 3.500 & 0.5000 & 3.390 & 0.5008 & 2.5690 & 4.2134 \\
\hline$\xi_{w}$ & bêta & 0.750 & 0.0500 & 0.760 & 0.0343 & 0.7064 & 0.8175 \\
\hline$\xi_{p}$ & bêta & 0.750 & 0.0500 & 0.851 & 0.0194 & 0.8190 & 0.8826 \\
\hline$\gamma_{w}$ & bêta & 0.250 & 0.1500 & 0.161 & 0.1039 & 0.0085 & 0.3092 \\
\hline$\gamma_{p}$ & bêta & 0.250 & 0.1500 & 0.915 & 0.0344 & 0.8627 & 0.9696 \\
\hline$\varphi^{-1}$ & normale & 5.500 & 1.5000 & 8.529 & 1.1758 & 6.5938 & 10.4514 \\
\hline$h$ & bêta & 0.500 & 0.1500 & 0.784 & 0.0606 & 0.6917 & 0.8806 \\
\hline$\rho$ & bêta & 0.800 & 0.0500 & 0.889 & 0.0226 & 0.8537 & 0.9258 \\
\hline$r_{\pi}$ & normale & 1.700 & 0.1000 & 1.654 & 0.0995 & 1.4910 & 1.8181 \\
\hline$r_{y}$ & normale & 0.125 & 0.0500 & 0.061 & 0.0372 & 0.0003 & 0.1205 \\
\hline$r_{\Delta \pi}$ & normale & 0.300 & 0.1000 & 0.376 & 0.0984 & 0.2147 & 0.5369 \\
\hline$r_{\Delta y}$ & normale & 0.062 & 0.0500 & 0.002 & 0.0076 & -0.0106 & 0.0138 \\
\hline$\rho_{G}$ & bêta & 0.800 & 0.1000 & 0.934 & 0.0309 & 0.8869 & 0.9808 \\
\hline$\rho_{\pi}$ & bêta & 0.800 & 0.1000 & 0.915 & 0.0511 & 0.8456 & 0.9886 \\
\hline$\zeta^{a}$ & uniforme & 0.500 & 0.2887 & 0.706 & 0.0510 & 0.6260 & 0.7927 \\
\hline$\zeta^{B}$ & bêta & 0.600 & 0.1000 & 0.443 & 0.0815 & 0.3113 & 0.5796 \\
\hline$\zeta^{L}$ & uniforme & 0.500 & 0.2887 & 0.220 & 0.1186 & 0.0075 & 0.3863 \\
\hline$\sigma_{R}$ & uniforme & 0.500 & 0.2887 & 0.095 & 0.0123 & 0.0757 & 0.1140 \\
\hline$\sigma_{\pi}$ & uniforme & 0.500 & 0.2887 & 0.215 & 0.0419 & 0.1473 & 0.2829 \\
\hline$\sigma_{G}$ & uniforme & 2.500 & 1.4434 & 1.858 & 0.1781 & 1.5710 & 2.1441 \\
\hline$\sigma_{a}$ & uniforme & 2.500 & 1.4434 & 0.924 & 0.0926 & 0.7739 & 1.0724 \\
\hline$\sigma_{L}$ & uniforme & 20.000 & 11.5470 & 22.323 & 5.5379 & 13.3526 & 31.2234 \\
\hline$\sigma_{B}$ & uniforme & 5.000 & 2.8868 & 5.483 & 1.3295 & 3.3360 & 7.5805 \\
\hline$\sigma_{I}$ & uniforme & 2.500 & 1.4434 & 1.705 & 0.1874 & 1.4002 & 2.0015 \\
\hline
\end{tabular}

TAB. 1 - Résultats du Metropolis-Hastings. L'intervalle $\mathcal{I}$ défini par la borne inférieure $\mathcal{I}_{1}$ et la borne supérieure $\mathcal{I}_{2}$ est le plus petit intervalle contenant $80 \%$ de la distribution postérieure.

un bruit blanc sur le choc de taux d'intérêt, $\eta_{t}^{R}$, qui suit une loi normale $\mathcal{N}\left(0, \sigma_{R}\right)$ et on suppose que $\varepsilon_{t}^{I} \equiv \mathcal{N}\left(0, \sigma_{I}\right)$. Elle se différencie de celle employée dans Smets et Wouters (2003) principalement par l'absence de choc sur le mark-up, dont l'interprétation structurelle peut être discutée.

Les espérances et écarts types postérieurs, ainsi que les Highest Probability Intervals (le plus petit intervalle contenant $80 \%$ de la distribution postérieure), sont reportés dans le tableau (1). Les densités postérieures sont représentées dans la figure 1. Les valeurs estimées des paramètres autorégressifs des chocs persistants s'étendent de 0,22 (choc d'offre de travail) à 0,93 (choc sur la cible d'inflation). Le choc d'offre de travail apparaît particulièrement volatil. Les moyennes postérieures des paramètres associés aux rigidités nominales sont $\xi_{w}=0,76$ et $\xi_{p}=0,85$. Les moyennes postérieures des paramètres d'indexation sont $\gamma_{w}=0.16$ et $\gamma_{p}=0.92$. L'indexation de l'inflation contemporaine sur l'inflation passée est proche de zéro, les données semblent très informatives dans cette direction ${ }^{14}$, contrairement aux estimations reportées par Smets et Wouters (2003). Les données sont bien moins informatives sur les élasticités $\sigma_{c}$ et $\sigma_{l}$ (voir les figures 1(h) et 1(i)). Les paramètres associés à l'inflation dans la règles de Taylor sont faiblement identifiés par les données, dans le sens où, par exemple, la distribution postérieure

14. Dans le sens où il y a une différence appréciable entre les variances a priori et a posteriori. Visuellement, la distribution postérieure de $\gamma_{p}$ (figure $1(\mathrm{~m})$ ) est beaucoup plus concentrée que sa distribution a priori. 
de $r_{\pi}$ est très proche de sa distribution a priori (figure $1(\mathrm{q})$ ).

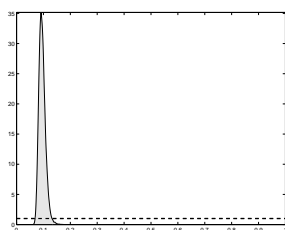

(a) $\sigma_{R}$

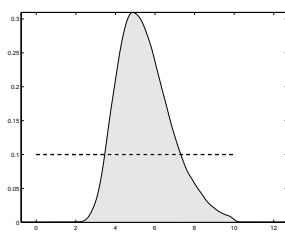

(f) $\sigma_{B}$

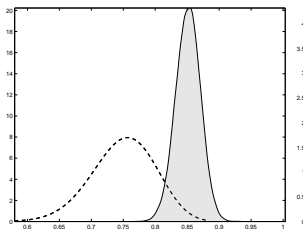

(k) $\xi_{p}$

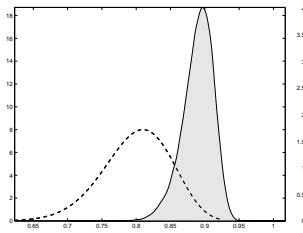

(p) $\rho$

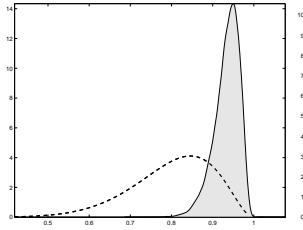

(u) $\rho_{g}$

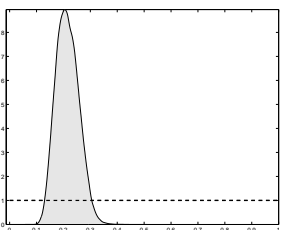

(b) $\sigma_{\pi}$

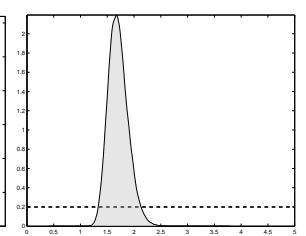

(g) $\sigma_{I}$

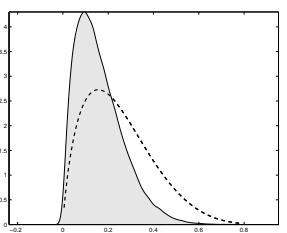

(1) $\gamma_{w}$

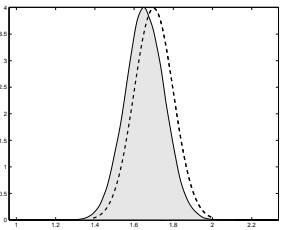

(q) $r_{\pi}$

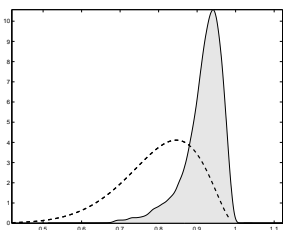

(v) $\rho_{\pi}$

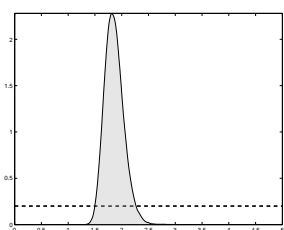

(c) $\sigma_{G}$

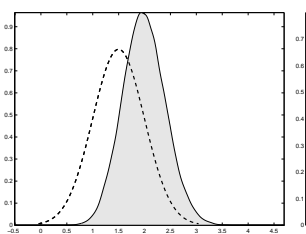

(h) $\sigma_{c}$

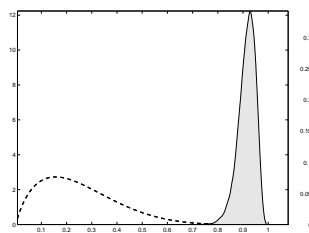

(m) $\gamma_{p}$

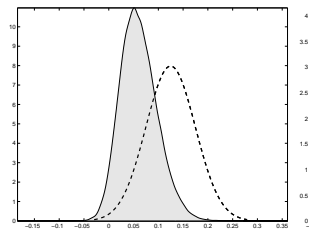

(r) $r_{y}$

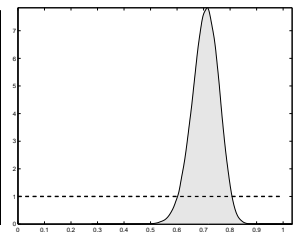

(w) $\zeta^{a}$

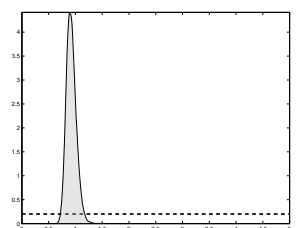

(d) $\sigma_{a}$

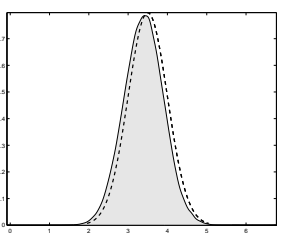

(i) $\sigma_{l}$

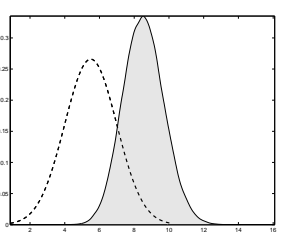

(n) $\varphi^{-1}$

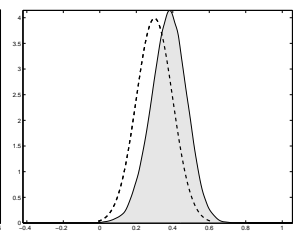

(s) $r_{\Delta \pi}$

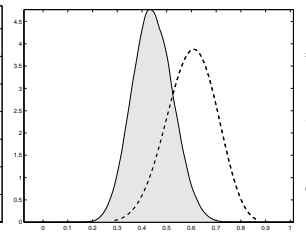

(x) $\zeta^{B}$

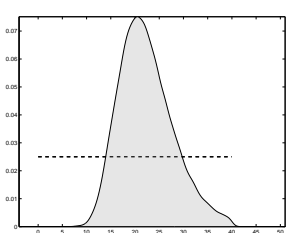

(e) $\sigma_{L}$

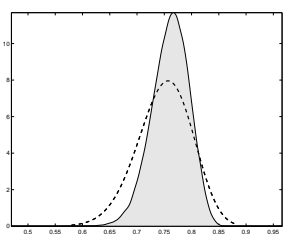

(j) $\xi_{w}$

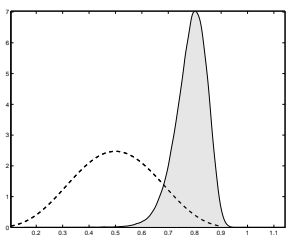

(o) $h$

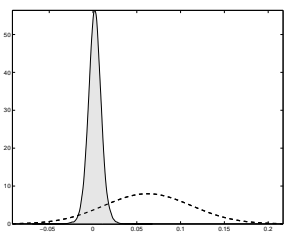

(t) $r_{\Delta y}$

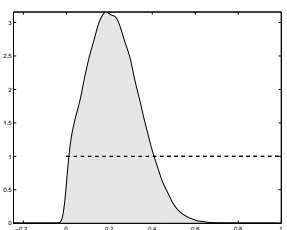

(y) $\zeta^{L}$

FIG. 1 - Densités a priori et a posteriori. Les courbes en tirets noirs représentent les densités a priori, les frontières des surfaces grisées représentent les densités a posteriori.

\section{Variantes}

\subsection{Caractérisation de l'incertitude}

Dans cette section, nous illustrons une des possibilités offertes par l'estimation de modèles structurels tournés vers le futur. Il s'agit de simuler la réponse du modèle à des chocs déterministes structurels ou de politique économique, qui éventuellement modifient l'état stationnaire. Dans la mesure où le niveau de long terme peut être affecté par ces chocs, un modèle stochastique résolu par une méthode de perturbation ne convient pas (voir Juillard et al. (2008)). Pour cette raison, nous abandonnons l'hypothèse 
d'anticipations rationnelles au profit de celle d'anticipations parfaites.

Nous envisageons un choc permanent anticipé sur la TVA. L'objet de cette section est d'illustrer comment nous pouvons projeter l'incertitude quant à la paramétrisation du modèle sur l'espace des variantes. Par exemple, nous désirons déterminer, étant donnée notre incertitude sur les paramètres du modèle (section 2), la probabilité que le salaire réel baisse lorsque les ménages et les firmes apprennent que la TVA augmentera de deux points deux ans plus tard. L'incertitude envisagée ici ne concerne que l'économiste ; nous supposons que les agents connaissent les paramètres du modèle.

L'incertitude est caractérisée par la densité postérieure, $p\left(\theta \mid \mathcal{Y}_{T}\right)$, obtenue à l'issue de l'estimation du modèle (section 3). Notons $\left\{v_{s}\right\}_{s=0}^{\mathcal{H}}$, une suite de vecteurs $m \times 1$, les trajectoires d'un ensemble de variables endogènes suite à l'annonce à la date $1 \mathrm{~d}$ 'un choc permanent à la période $s>1^{15}$. Pour $\theta$ donné, le vecteur regroupant les paramètres du modèle, on peut construire la suite $\left\{v_{s}\right\}_{s=1}^{\mathcal{H}}$, on notera $v_{s}=\Upsilon_{s}(\theta)$ pour tout $s \geq 0{ }^{16}$. Nous pouvons alors calculer la densité postérieure et les moments postérieurs de $\left\{v_{s}\right\}_{s=1}^{\mathcal{H}}$. Par exemple, l'espérance postérieure de $v_{s}$ est :

$$
\mathbb{E}\left[v_{s} \mid \mathcal{Y}_{T}\right]=\int_{\Theta} \Upsilon_{s}(\theta) p\left(\theta \mid \mathcal{Y}_{T}\right) \mathrm{d} \theta
$$

où $\Theta$ est l'espace des paramètres structurels. Plus généralement, la densité postérieure de $v_{s}$ est :

$$
\widetilde{p}\left(v_{s}\right)=\left|J_{\Upsilon_{s}}\right|^{-1} p\left(v_{s}\right)
$$

où $J_{\Upsilon_{s}}$ est la matrice jacobienne associée à $\Upsilon_{s}$. En pratique, nous utilisons les simulations issues du Metropolis-Hastings (MH), mis en œuvre dans la section 3 afin d'évaluer la distribution postérieure de $\theta$. On sélectionne $B$ vecteurs de paramètres structurels, $\left\{\theta^{(b)}\right\}_{b=1}^{B}$, en tirant uniformément dans les simulations du MH. L'espérance postérieure de $v_{s}$, par exemple, est alors estimée par:

$$
\widehat{\mathbb{E}} \widehat{\left[v_{s} \mid \mathcal{Y}_{T}\right]}=\frac{1}{B} \sum_{b=1}^{B} \Upsilon_{s}\left(\theta^{(b)}\right)
$$

Pour chaque vecteur de paramètres structurels, $\theta^{(b)}$, on résoud le modèle à anticipation parfaite et on reporte la moyenne empirique des trajectoires obtenues ${ }^{17}$.

En pratique, nous devons faire quelques choix. Certains paramètres estimés affectent l'état stationnaire. Dans notre cas, les paramètres $h$, le degré d'habitude dans les choix de consommation, $\sigma_{c}$, l'élasticité

15. À la date zéro, les variables sont initialisées à l'état stationnaire.

16. La fonction $\Upsilon_{s}$ résume l'algorithme de relaxation utilisé pour résoudre le modèle à anticipations parfaites.

17. La figure 2 est obtenue avec $B=2000$. La courbe noire représente la moyenne (postérieure) des trajectoires. Chaque courbe grise représente une trajectoire correspondant à un vecteur de paramètres structurels. 
intertemporelle de la consommation, et $\sigma_{l}$, l'élasticité de l'effort travail, ont une influence sur l'état stationnaire. Or notre exercice de variante est initialisé (en $s=0$ ) à l'état stationnaire qui prévaut avant l'annonce (en $s=1$ ) du choc fiscal. Ainsi, en considérant l'incertitude sur l'ensemble des paramètres estimés nous obtiendrions une distribution sur l'état stationnaire, et par conséquent sur le point initial. Cette propriété peut paraître peu désirable, car elle complique l'interprétation des variantes. Nous avons donc choisi de ne pas considérer l'incertitude sur ces trois paramètres ${ }^{18}$. Une alternative serait de représenter les taux de croissance des variables plutôt que les niveaux, puisqu' aucun paramètre n'affecte le taux de croissance de long terme, afin d'étudier la dynamique de l'économie suite à l'annonce d'un choc de politique fiscale.

\subsection{Choc de TVA}

Nous présentons dans cette section les réponses de long et court termes du modèle à une hausse permanente anticipée du taux de TVA $\left(\tau^{C}-1\right)$ de deux points. À long terme, le choc fiscal induit une réduction de la consommation, même si l'augmentation de la recette fiscale est intégralement reversée sous forme forfaitaire aux ménages. En effet, il crée une distorsion du prix de la consommation par rapport au coût d'opportunité du loisir. Ainsi, la hausse de TVA modifie les arbitrages du ménage en faveur du loisir. À long terme, le choc fiscal détériore l'emploi, le stock de capital (les facteurs de production sont imparfaitement complémentaires) et le produit. Au final, l'augmentation de la pression fiscale coûte $0,3 \%$ du PIB.

Le choc fiscal est annoncé à la date Q1 (trait rouge plein sur la figure 2) et intervient effectivement à la fin de Q8 (trait rouge en pointillés sur la figure 2). L'annonce du choc de TVA modifie l'arbitrage intertemporel entre consommation contemporaine et consommation future ; les ménages, prévoyant une augmentation du prix relatif de la consommation, choisissent d'ajuster continument à la hausse la consommation jusqu'à la date du choc (figure 2(a)). Cet ajustement est limité par la présence d'habitudes de consommation. En moyenne, l'annonce en Q1 induit un saut de $0,1 \%$ de la consommation. Juste avant la réalisation du choc, en Q8, l'augmentation cumulée est de 0,4\% par rapport à l'état stationnaire initial. Par la suite, la consommation baisse rapidement pour rejoindre son nouvel état stationnnaire : au douxième trimestre, elle a déjà retrouvé son niveau d'avant l'annonce et dix ans après, en Q40, elle a perdu 0,3\%. Il convient de noter que l'incertitude sur le modèle ne se traduit que marginalement par une incertitude quant à la réaction de la consommation. Ainsi les trajectoires obtenues pour différentes valeurs des paramètres structurels sont très proches. Graphiquement, sur la figure 2(a), on observe que la surface grisée est très concentrée autour de la moyenne postérieure. Ceci s'explique par le fait que les paramètres $h$ et $\sigma_{c}$ de la courbe IS - equation 2 - sont fixés dans cet exercice.

18. Ils sont étalonnés à l'espérance postérieure. 
Pour financer ce besoin de consommation supplémentaire, chaque ménage est incité à augmenter son salaire nominal dès qu'il en a la possibilité. En conséquence, le salaire nominal augmente entre la date de l'annonce du choc et celle de son intervention. En moyenne, ceci se retrouve dans l'évolution à la hausse du salaire réel (figure 2(b)), qui, au moment de la réalisation du choc, atteint un niveau supérieur de $0,1 \%$ à son niveau inital. Cependant, l'effet à la date de l'annonce est plus ambigu. Sur la figure 2(b), on observe que selon les valeurs des paramètres structurels, le salaire réel peut augmenter ou chuter lorsque les ménages apprennent la hausse future de TVA. La probabilité postérieure d'un saut à la baisse est de 38,6\%. La figure (3) représente (la courbe noire) un estimateur à noyau de la densité du salaire réel au moment de l'annonce. Le trait vertical rouge représente la condition initiale (l'état stationnaire) du salaire réel. Cette ambiguïté est liée à l'incertitude associée au paramètre de Calvo sur les salaires, $\xi_{w}$. Envisageons deux scenarii polaires :

(i) $\mathrm{Si} \xi_{w}$ est proche de 1, les ménages ne peuvent pas ajuster leurs salaires nominaux au moment de l'annonce. Par ailleurs, les firmes, qui anticipent simultanément une hausse de leur coût marginal, augmentent dès que possible leur prix, ce qui se traduit par une hausse instantanée de l'inflation dès l'annonce du choc (figure 2(c)). Cela entraîne une baisse du salaire réel en Q1.

(ii) $\mathrm{Si} \xi_{w}$ est proche de 0 , les ménages peuvent ajuster à la hausse leurs salaires nominaux au moment de l'annonce de façon à augmenter leur pouvoir d'achat. Ceci entraîne une augmentation en Q1 du salaire réel.

L'autorité monétaire réagit à la hausse de l'inflation en augmentant le taux d'intérêt nominal (Figure 2(d)). Cette réaction a pour corollaire d'augmenter le taux d'intérêt réel, ce qui amoindrit la hausse de la consommation.

Les ménages, en cherchant à augmenter leur consommation avant le choc, sont amenés à consommer leur capital. De plus, le niveau de capital productif nécessaire après la réalisation du choc est plus faible (la consommation des ménages baisse à long terme), ce qui renforce la baisse de l'investissement (-0,1\% en Q1) contrôlée par la présence d'un coût d'ajustement sur l'investissement (figure 2(e)). En moyenne, la somme de la consommation et de l'investissement (la demande des ménages) augmente de 0,04\% en Q1. Pour alimenter cet accroissement de demande, les ménages pourraient offrir une quantité supérieure de travail, mais $(i)$ cela détériorerait leur utilité et ne va pas dans le sens de l'arbitrage consommation loisir, (ii) par ailleurs les firmes sont plutôt incitées à réduire leur demande de travail suite à la hausse du coût du travail. La seule possibilité offerte aux ménages est de réduire le taux d'utilisation des capacités de production. En effet, une baisse de $z_{t}$ induit une aubaine $\psi\left(z_{t}\right) K_{t-1}$ (voir l'équation (23) d'équilibre sur le marché des biens). Cette baisse est limitée par la détérioration des revenus du capital des ménages 
(voir la condition nécessaire d'optimalité (5) qui impose l'égalisation du gain marginal et de la perte marginale associés à $z$ ). Cette aubaine est partiellement consommée puisqu'elle s'accompagne instantanément d'une baisse du produit (figure 2(f)). La baisse en Q1 de celui-ci a deux sources ${ }^{19}$ : $(i)$ le capital utilisé $\tilde{K}_{t}=z_{t} K_{t}$ baisse (figure 2(h)) et (ii) la frontière des prix des facteurs (14) indique que l'emploi doit s'ajuster à la baisse (figure 2(g)). La consommation augmentant régulièrement entre Q1 et Q8, et le stock de capital physique se réduisant sur tout l'exercice, dès Q2, les ménages réajustent à la hausse le taux d'utilisation du capital de façon à augmenter le stock de capital physique installé. Puisque la technologie est à facteurs imparfaitement complémentaires, cette évolution s'accompagne d'une remontée de la demande de travail. En moyenne, le produit augmente de 0,1\% entre Q1 et Q8.

Pour finir, le graphique 2(i) permet de compléter notre compréhension des effets du choc de TVA. L'écart de production augmente dès l'annonce de la réforme fiscale. Ce résultat est attendu, puisque cette variable mesure la distance entre le produit observé et le produit que nous observerions dans un monde sans rigidité nominale. En l'absence de rigidité sur les prix, la hausse initale du salaire réel est plus prononcée, ce qui se traduit via la frontière de prix des facteurs par une baisse de la demande de travail $L$ plus forte. Finalement, la réaction initiale de la production est plus marquée. Pour l'autorité monétaire, il n'y a pas d'arbitrage entre inflation et l'écart de production face à un choc anticipé de TVA: la dynamique de l'écart de production renforce la nécessité d'une politique monétaire restrictive.

19. Cette baisse est limitée par la présence du coût fixe, $\Phi$. En effet, celui-ci est linéaire dans le niveau de long terme du produit qui baisse dès l'annonce du choc fiscale. 


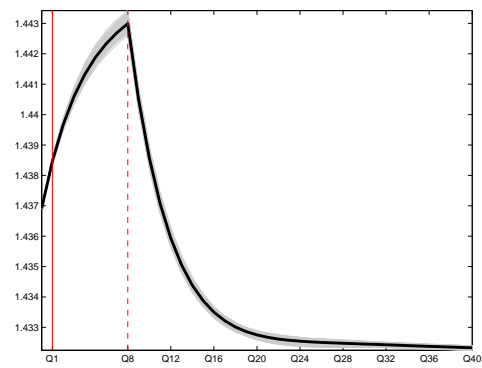

(a) Consommation

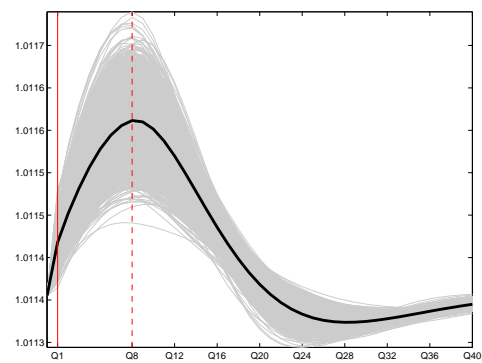

(d) Taux d'intérêt nominal

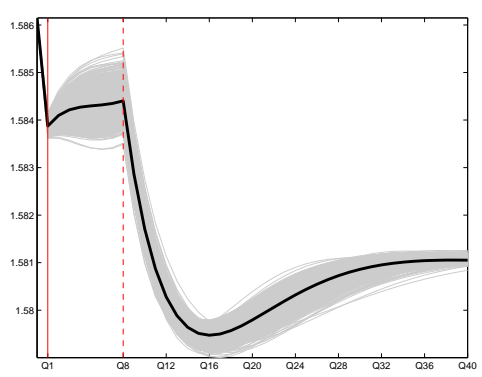

(g) Emploi

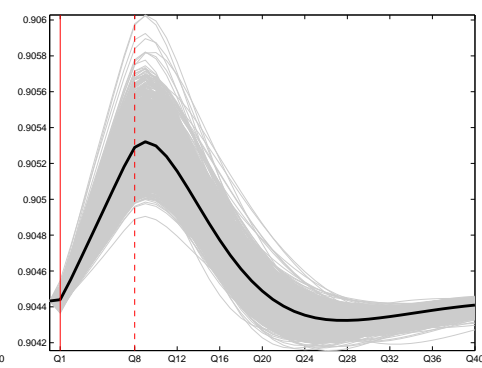

(b) Salaire réel

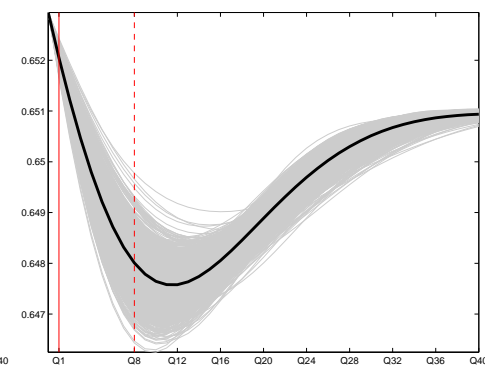

(e) Investissement

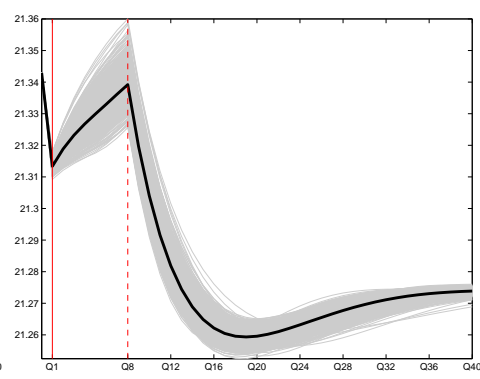

(h) Capital utilisé

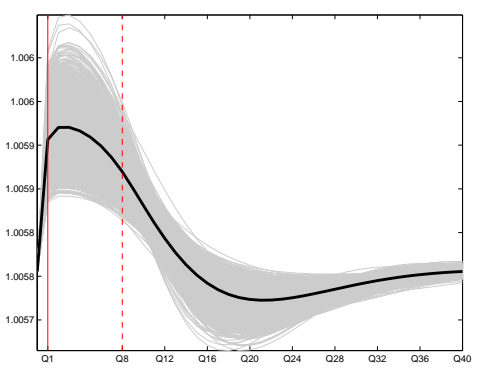

(c) Inflation

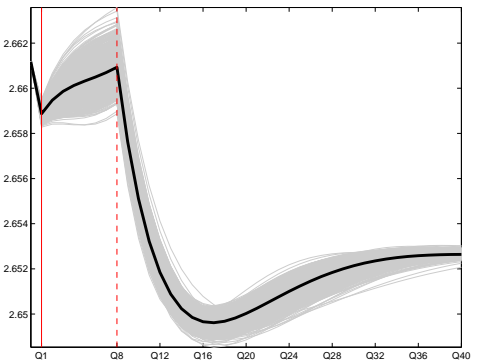

(f) Production

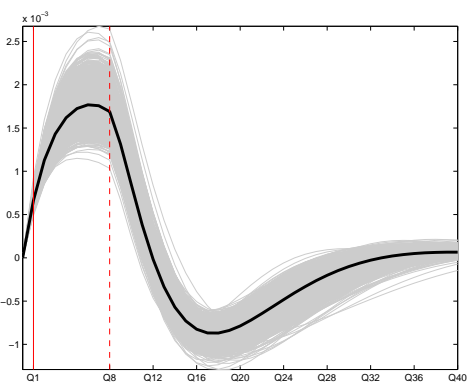

(i) Écart de production

FIG. 2 - Effets d'une hausse permanente et anticipée de TVA de 2 points. 


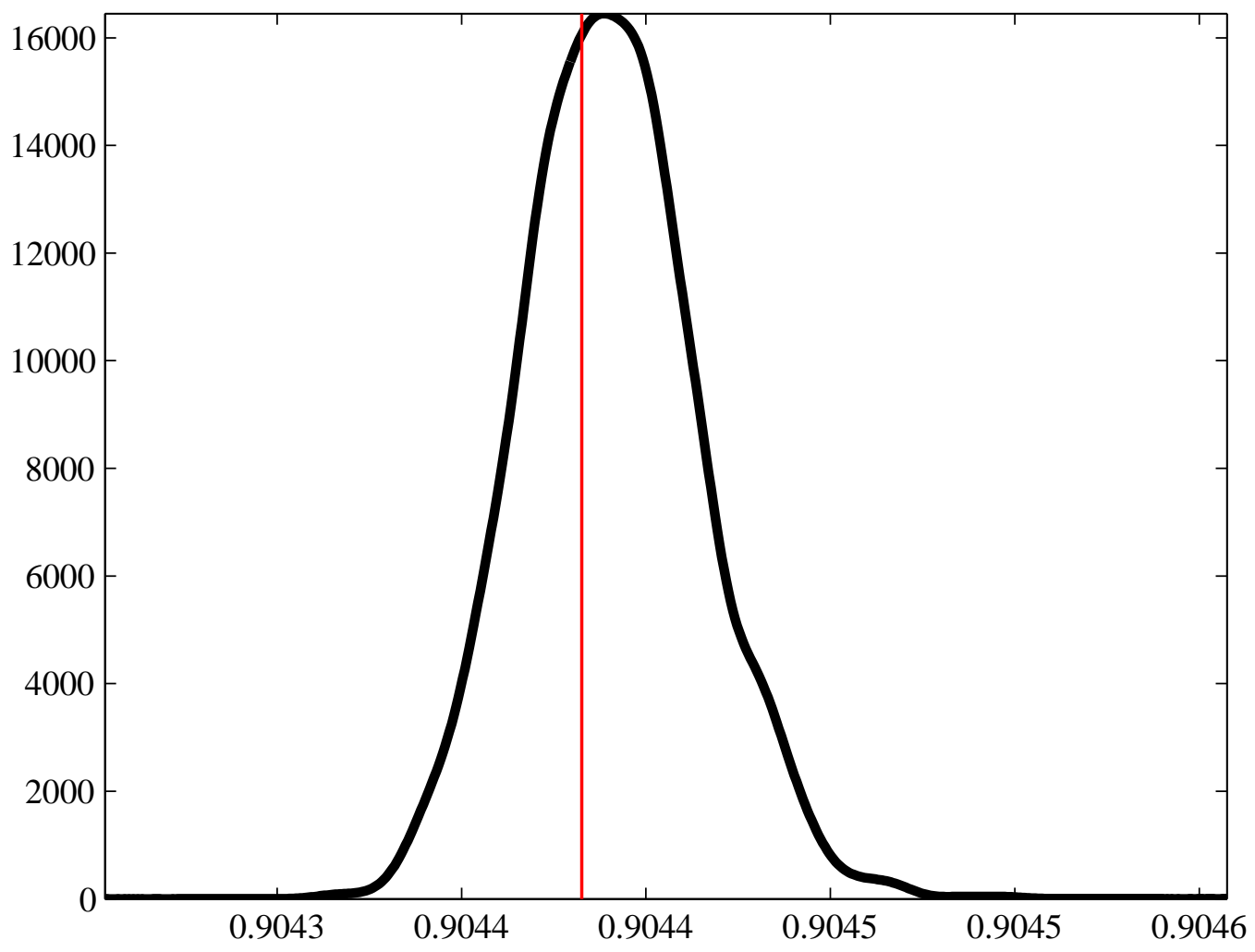

FIG. 3 - Impact de la politique fiscale sur le salaire réel, au moment de l'annonce. Ce graphique représente la densité de probabilité du salaire réel en Tl au moment de l'annonce. Celle-ci est estimée à l'aide d'un estimateur à noyau; nous avons utilisé une fenêtre gaussienne et choisi le paramètre de lissage à l'aide de la méthode de Sheather et Jones.

\section{Conclusion}

Nous avons illustré en quoi un regard bayésien sur les modèles d'équilibre général intertemporels stochastiques peut se révéler pertinent pour l'analyse de politiques économiques. L'estimation bayésienne d'un modèle DSGE, nous permet de rendre compte de l'incertitude sur les variantes construites à partir d'une version à anticipation parfaite du même modèle.

Il convient de souligner certaines limites de l'exercice considéré ici. Nous avons pris le parti de rester le plus proche possible de l'article de Smets et Wouters (2003) qui est à l'origine de l'intérêt du monde institutionnel pour les modèles DSGE. La variante envisagée ici, un choc anticipé sur le taux de TVA, serait sûrement plus riche d'enseignements dans un modèle où on distinguerait deux types de ménages : des individus ricardiens (l'hypothèse adoptée ici) et une proportion de ménages non ricardiens - qui consom- 
meraient la totalité de leur revenu salarial. Cette extension permettrait, par exemple, de s'interroger sur la contrepartie de la politique fiscale (que peut faire l'État des recettes fiscales supplémentaires?) tout en identifiant des effets supplémentaires d'une hausse de la TVA ${ }^{20}$.

La démarche poursuivie ici pourrait être étendue dans d'autres directions. D'abord sur la caractérisation de l'incertitude. Nous avons supposé que l'incertitude quant au DGP ne porte que sur les paramètres d'un modèle. C'est pourquoi nous n'avons estimé qu'un seul modèle. Nous pourrions élargir l'incertitude en supposant que le DGP est un mélange de modèles paramétrés, à mesure des densités marginales associées $^{21}$ (voir Adjemian et al. (2008)). Une seconde piste concerne l'initialisation de l'exercice de variante. Plutôt que d'initialiser la variante à un état stationnaire, nous pourrions utiliser une condition historique. L'exercice s'interpréterait alors comme une prévision conditionnelle. Par exemple, nous pourrions faire une prévision du PIB sachant que dans un an l'État va changer le taux de TVA. Ces prolongements seront développés dans des recherches ultérieures.

\section{Références}

Adjemian, S., Pelgrin, F. (2008). "Un regard bayésien sur les modèles dynamiques de la macroéconomie". Économie\& Prévision, n 183/184, 127-152.

Baghli, M., Brunhes-Lesage, V., De Bandt, O., Fraisse, H., Villetelle, J.-P. (2004). "Modèle d'Analyse et de préviSion de la COnjoncture TrimesTriellE". Notes d'Études et de Recherche, Banque de France, $\mathrm{n}^{\circ} 106$.

Calvo, G. (1983). "Staggered Prices in a Utility Maximizing Framework". Journal of Monetary Economics, 12, 383-398.

Christiano, L.J., Eichenbaum, M., Evans, C.L. (2005). "Nominal Rigidities and the Dynamic Effects of a Shock to Monetary Policy". Journal of Political Economy, 113(1), 1-45.

Coenen, G., Straub, R. (2004). "Non-Ricardian Households and Fiscal Policy in an Estimated DSGE Model of the Euro Area". mimeo.

Ecerg, C., Henderson, D., Levin, A. (2000). "Optimal Monetary Policy with Staggered Wage and Price Contracts". Journal of Monetary Economics, 46, 281-313.

Fève, P. (2005). "La modélisation macro-économétrique dynamique". Notes d'Études et de Recherche, Banque de France, $n^{\circ} 129$.

Juillard, M., Ocaktan, T. (2008). "Méthodes de simulation des modèles stochastiques d'équilibre général”. Économie\& Prévision, n 183/184, 115-126.

Lucas, R. (1976). "Econometric Policy Evaluation: a Critique". Canergie Rochester Conference Series on Public Policy, K. Brunner et A. Meltzer (Eds), 19-46.

Smets, F., Wouters, R. (2003). "An Estimated Dynamic Stochastic General Equilibrium Model of the Euro Area". Journal of the European Economic Association, 1(5), 1123-1175.

20. Voir, par exemple, Coenen et al. (2004) qui estiment un tel modèle.

21. La densité marginale mesure la qualité d'ajustement d'un modèle. 


\section{Documents de Travail}

229. Ph. Aghion, Ph. Askenazy, R. Bourlès, G. Cette and N. Dromel, "Education, Market Rigidities and Growth,” January 2009

230. G. Cette and M. de Jong, "The Rocky Ride of Break-even-inflation rates," January 2009

231 E. Gautier and H. Le Bihan, "Time-varying $(S, s)$ band models: empirical properties and interpretation," January 2009

232. K. Barhoumi, O. Darné and L. Ferrara, "Are disaggregate data useful for factor analysis in forecasting French GDP ? " February 2009

233 R. Cooper, H. Kempf and D. Peled, "Monetary rules and the spillover of regional fiscal policies in a federation" February 2009

234 C. Jardet, A. Monfort, and F. Pegoraro, "No-arbitrage Near-Cointegrated VAR(p) Term Structure Models, Term Premia and GDP Growth" June 2009

235 C. Jardet, A. Monfort, and F. Pegoraro, "New Information Response Functions," June 2009

236 S. Adjemian, C. Cahn, A. Devulder et N. Maggiar, «Variantes en Univers Incertain », Juin 2009

Pour accéder à la liste complète des Documents de Travail publiés par la Banque de France veuillez consulter le site : http://www.banque-france.fr/fr/publications/publications.htm

For a complete list of Working Papers published by the Banque de France, please visit the website: http://www.banque-france.fr/gb/publications/publications.htm

Pour tous commentaires ou demandes sur les Documents de Travail, contacter la bibliothèque de la Direction Générale des Études et des Relations Internationales à l'adresse suivante :

For any comment or enquiries on the Working Papers, contact the library of the Directorate General Economics and International Relations at the following address :

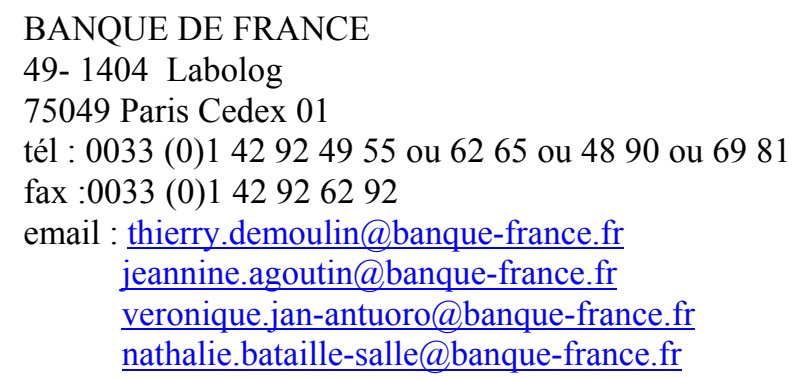

